\title{
circ_0067934 increases bladder cancer cell proliferation, migration and invasion through suppressing miR-1304 expression and increasing Myc expression levels
}

\author{
QIAN LIU $^{1 *}$, QI ZHOU ${ }^{2 *}$ and PENG ZHONG ${ }^{2}$ \\ ${ }^{1}$ Department of Oncology, Puren Hospital, Wuhan University of Science and Technology, Wuhan, Hubei 430081; \\ ${ }^{2}$ Department of Urology, Hanchuan People's Hospital, Hanchuan, Hubei 431600, P.R. China
}

Received February 13, 2019; Accepted February 7, 2020

DOI: $10.3892 /$ etm.2020.8648

\begin{abstract}
RNAs have been demonstrated to be key regulators of bladder cancer progression. The present study aimed to investigate the effects of circular RNA (circ)_0067934 in bladder cancer progression. A total of 54 patients with primary bladder cancer were enrolled, and their tumor tissues and adjacent normal bladder tissues were collected. For in vitro functional assays, T24 cells were transfected with sicirc_0067934, and Cell Counting Kit-8 was used to analyze the proliferative capacity of T24 cells. In addition, Transwell and Matrigel assays were used to assess the cell migration and invasion abilities, and a dual-luciferase reporter assay was used to investigate the relationship between miR-1304 and circ_0067934. Finally, reverse transcription-quantitative PCR and western blotting were performed to analyze gene and protein expression levels, respectively. circ_0067934 expression levels were significantly increased in bladder cancer tissues $(\mathrm{P}<0.001)$, which was associated with metastasis and a significantly decreased 5-year overall $(\mathrm{P}<0.05)$ and disease-free survival $(\mathrm{P}<0.05)$. In vitro, $\mathrm{T} 24$ cells in the small interfering RNA (si)circ_0067934 group demonstrated significantly reduced proliferation, migration and invasion abilities compared with the si negative control (siNC) group $(\mathrm{P}<0.01)$. In addition, the knockdown of circ_0067934 directly increased microRNA (miR)-1304 expression levels in T24 cells. Myc was subsequently discovered to be directly inhibited by miR-1304 and circ_0067934 was observed to increase Myc expression levels in T24 cells through inhibiting miR-1304 expression levels $(\mathrm{P}<0.01)$. Compared with the
\end{abstract}

Correspondence to: Professor Peng Zhong, Department of Urology, Hanchuan People's Hospital, 1 People's Avenue, Hanchuan, Hubei 431600, P.R. China

E-mail: pengzhong121@sina.com

*Contributed equally

Key words: circular RNA_0067934,bladder cancer,microRNA-1304, Myc, proliferation
siNC group and sicirc_0067934 + Myc overexpression group, T24 cells in the sicirc_0067934 group exhibited significantly decreased proliferative, migratory and invasive abilities $(\mathrm{P}<0.01)$. In conclusion, circ_0067934 was demonstrated to increase bladder cancer cell proliferation, migration and invasion through promoting Myc expression levels via the suppression of miR-1304 expression.

\section{Introduction}

Bladder cancer is a common malignant tumor of the urinary system, which is highly recurrent in nature, and demonstrates a high infiltration rate and a strong tendency to metastasize $(1,2)$. The incidence rate of bladder cancer ranks it as the eleventh most common malignant tumor worldwide and the sixth amongst male malignant tumors; its incidence rate increases with age and invasive metastatic bladder cancer leads to higher rates of mortality $(1,2)$. At present, research into the pathogenesis, prevention, early diagnosis and treatment of bladder cancer remains in its infancy. Thus, investigating the molecular mechanisms of its pathogenesis may provide novel strategies for the diagnosis and treatment of bladder cancer.

Previous studies over the past few decades have demonstrated that most mammalian cells are capable of transcriptionally producing short or long RNAs that lack protein coding ability $(3,4)$. Circular RNAs (circ/circRNAs) are a relatively newly discovered type of non-coding RNA, which are abundant in number and highly stable in nature due to their covalent closed-loop structure (4). The expression of circRNAs is tissue-specific, and most circRNAs exhibit differential expression patterns in different species, tissues and cells $(5,6)$. The majority of circRNAs are $<1,500$ nucleotides in length, with an average length of 500 nucleotides $(7,8)$. Several circRNAs have been identified to be closely related to the recurrence and prognosis of bladder cancer. For example, circRNA myosin light chain kinase, circRNA cerebellar degeneration related protein 1 antisense and circRNA homeodomain interacting protein kinase 3 have all been demonstrated to serve an important role in the occurrence, development and metastasis of bladder cancer (9-11); these circRNAs all participated in important biological roles, such as the proliferation, apoptosis, migration and invasion 
of bladder cancer, by regulating epigenetic modifications and important cell signaling transduction pathways. In addition, in multiple studies, circ_0067934 has been reported to serve as an oncogene in several types of tumor $(12,13)$. In the present study, the expression levels of circ_006734 were also discovered to be increased in bladder cancer tissues. Thus, the impact of circ_0067934 on the progression of bladder cancer was subsequently investigated. To the best of our knowledge, this is the first study to report on the effects of circ_0067934 in bladder cancer.

\section{Materials and methods}

Patient studies. From July 2011 to September 2013, 54 bladder cancer tissues (40 males and 14 females; age range, 35-68 years old; median age, 54 years old; 34 metastasis and 20 non-metastasis) were collected from Hanchuan People's Hospital. Patients who were treated with radiotherapy or chemotherapy before surgery were excluded. All patients voluntarily joined the study and written informed consent was obtained. The study was approved by the Ethics Committee of Hanchuan People's Hospital. The association between clinicopathological features and circ_0067934 expression levels in 54 bladder cancer tissues is presented in Table I.

During surgery, all patients' tumor tissues and adjacent normal bladder tissues were collected and 5 years of follow-up was completed. During the 5 years, the follow-up endpoint was the time of death. The effects of circ_0067934 expression levels on metastasis, 5-year overall survival and disease-free survival were subsequently analyzed.

Cell lines and reagents. The human bladder epithelial SV-HUC-1 cell line and bladder cancer cell lines, T24, RT4 and UMUC3, were purchased from the Kunming Institute of Zoology, Chinese Academy of Sciences. All cells were cultured in DMEM (Invitrogen; Thermo Fisher Scientific, Inc.), supplemented with 10\% FBS (Thermo Fisher Scientific, Inc.), $1,000 \mathrm{IU} / \mathrm{ml}$ penicillin (Beijing Solarbio Science \& Technology Co., Ltd.) and $100 \mathrm{mg} / \mathrm{ml}$ streptomycin (Beijing Solarbio Science \& Technology Co., Ltd.). Cells were maintained at $37^{\circ} \mathrm{C}$ and $5 \% \mathrm{CO}_{2}$ in a humid environment.

Cell transfection and experimental groupings. T24 cells in DMEM without FBS and antibiotics were plated into six-well plates at $37^{\circ} \mathrm{C}$ and $5 \% \mathrm{CO}_{2}$ in a humid environment. Upon cells reaching $70 \%$ confluence, Lipofectamine ${ }^{\circledR} 2000$ reagent (Thermo Fisher Scientific, Inc.) was used for all transfections, according to the manufacturer's protocol. All plasmids were transfected at the concentration of $100 \mathrm{nM}$. The following molecules, which were all obtained from Shanghai GenePharma Co., Ltd., were used: circ_0067934 small interfering (si)RNA (sicirc_0067934; 5'-UGUUGAUUGGGAUAU GUUAUU-3'; $100 \mathrm{nM}$ ) and its negative control (siNC; 5'-GAA UGCUCCGUA AUCUGAACC-3'; $100 \mathrm{nM}$ ), microRNA (miRNA/miR)-1304 mimic (5'-UUUGAGGCUACAGUG AGAUGUG-3'; $100 \mathrm{nM}$ ) and its NC (miR-NC mimic ; 5'-UCA CAACCUCCUAGAAAGAGUAGA-3'; $100 \mathrm{nM}$ ), miR-1304 inhibitor (5'-CACAUC UCACUGUAGCCUCAA A-3'; $100 \mathrm{nM}$ ) and its NC (miR-NC inhibitor; 5'-GCGUAACUA AUACAUCGGAUUCGU-3'; $100 \mathrm{nM}$ ), Myc overexpression vector (oeMyc) and its $\mathrm{NC}$ expression vector (oeVector). The myc coding sequence was inserted into pcDNA3 vector (Invitrogen; Thermo Fisher Scientific, Inc.) to generate the oeMyc plasmid. The empty vector (oeVector) was used as a negative control.

A period of $48 \mathrm{~h}$ after transfection, the efficiency was validated by RT-qPCR and cells were used for the following experiments.

Cell Counting Kit-8 (CKK-8) assay. Following transfection, $2 \times 10^{3} \mathrm{~T} 24$ cells/well were seeded into 96 -well plates (4 replicates/group). DMEM, supplemented with $10 \%$ FBS, was added into each well at a volume of $200 \mu \mathrm{l}$ and cells were incubated at $37^{\circ} \mathrm{C}$ and $5 \% \mathrm{CO}_{2}$ in a humid environment. According to the manufacturer's protocol, $10 \mu \mathrm{l}$ CCK-8 reagent (Beijing Transgen Biotech Co., Ltd.) was added to each well following 24, 48, 72 and $96 \mathrm{~h}$ of incubation and incubated for a further $2 \mathrm{~h}$ at room temperature. The optical density value of each well at a wavelength of $450 \mathrm{~nm}$ was measured using an ELx808 microplate reader (BioTek Instruments, Inc.).

Migration and invasion assay. Following transfection, $2 \times 10^{5}$ T24 cells/well ( 3 replicates/group) were plated into the upper chambers of 24-well Transwell plates in $200 \mu 1$ serum-free DMEM. Transwell membranes were precoated with Matrigel (BD Biosciences) for $30 \mathrm{~min}$ at $37^{\circ} \mathrm{C}$. A volume of $500 \mu \mathrm{l}$ DMEM, supplemented with $10 \%$ FBS, was plated in the lower chambers. Following incubation at $37^{\circ} \mathrm{C}$ and $5 \% \mathrm{CO}_{2}$ for $24 \mathrm{~h}$, the Transwell chamber was removed and non-invasive cells were carefully removed using a cotton swab. Invasive cells were fixed in $95 \%$ ethanol for $5 \mathrm{~min}$ at room temperature and stained with $0.1 \%$ crystal violet for $10 \mathrm{~min}$ at room temperature. Stained cells were counted in five randomly selected fields using an inverted light microscope (magnification, x100). The migratory ability of the cells was investigated using the same method as aforementioned; however, the upper chamber was not coated with Matrigel.

Dual-luciferase reporter assay. The regulatory relationship between circ_0067934 and miR-1304 or between miR-1304 and Myc was verified using dual-luciferase reporter assays. Briefly, the 3'-untranslated region (UTR) binding sites were predicted using the following online prediction tools: miRDB database (http://mirdb.org/miRBD/index. html) and TargetScan7 (http://www.targetscan.org/vert_71). The circ_0067934-mutant (Mut) 3'-UTR sequence and circ_0067934 wild-type (WT) 3'-UTR sequence, as well as the Myc-Mut 3'-UTR sequence and Myc-WT 3'-UTR sequence were subsequently designed and cloned into the pGL3 vector (Promega Corporation). T24 cells $\left(2 \times 10^{3}\right.$ cell per well) were co-transfected with each of these vectors and either the miR-NC mimic or the miR-1304 mimic using Lipofectamine ${ }^{\circledR}$ 2000 reagent (Thermo Fisher Scientific, Inc.). Following incubation for $48 \mathrm{~h}$ at $37^{\circ} \mathrm{C}$ and $5 \% \mathrm{CO}_{2}$, the relative luciferase activities were detected using a Dual-Luciferase Reporter assay kit (Promega Corporation) according to the manufacturer's protocol and normalized to Renilla luciferase activity.

Reverse transcription-quantitative PCR (RT-qPCR). Total RNA in tissues and cells (following transfection for $48 \mathrm{~h}$ ) was 
Table I. Association between clinicopatholigcal features and circular RNA_0067934 expression levels in 54 bladder cancer tissues.

\begin{tabular}{lrcc}
\hline Variable & Low $(\mathrm{n}=26)$ & High $(\mathrm{n}=28)$ & P-value \\
\hline Age, years & & & 0.418 \\
$\quad<60$ & 17 & 15 & \\
$\geq 60$ & 9 & & \\
Sex, $\mathrm{n}$ & & 19 & 0.358 \\
$\quad$ Male & 21 & 9 & \\
Female & 5 & & 0.014 \\
Tumor size, cm & & 12 & \\
$<3$ & 20 & 16 & \\
$\geq 3$ & 6 & & 0.002 \\
Tumor stage & & 7 & \\
Ta-T1 & 18 & 21 & \\
T2-T4 & 8 & & \\
Lymph node & & 5 & \\
metastasis & & 23 & \\
No & 15 & & \\
Yes & 11 & & \\
\hline
\end{tabular}

extracted using TRIzol ${ }^{\circledR}$ reagent (Invitrogen; Thermo Fisher Scientific, Inc.), according to the manufacturer's protocol. A total of $500 \mu \mathrm{g}$ RNA was reverse transcribed into cDNA using the PrimeScript ${ }^{\mathrm{TM}}$ RT reagent kit (Takara Biotechnology Co., Ltd.), according to the manufacturer's protocol. PCR was subsequently performed using the SYBR Green I Supermix (Takara Biotechnology Co., Ltd.), according to the manufacturer's protocol using an iCycler IQ multicolor Detection System (Bio-Rad Laboratories, Inc.). The following thermocycling conditions were used for the qPCR: Initial denaturation at $94^{\circ} \mathrm{C}$ for $2 \mathrm{~min}$; and 40 cycles at $94^{\circ} \mathrm{C}$ for $30 \mathrm{sec}$ and $60^{\circ} \mathrm{C}$ for $30 \mathrm{sec}$. Expression levels were determined using the $2^{-\Delta \Delta \mathrm{Cq}}$ method (14) and normalized to the internal reference genes U6 or GAPDH. The primer pairs used were as follows: circ_0067934 forward, 5'-TAGCAGTTCCCCAATCCTTG-3' and reverse, 5'-CACAAATTCCCATCATTCCC-3'; miR-1304 forward, 5'-CGCGGTGTAGAGTGACATCG-3' and reverse, 5'-AGTGCAGGGTCCGAGGTATT-3'; U6 forward, 5'-GCT TCGGCAGCACATATACTAAAAT-3' and reverse, 5'-CGC TTCACGAATTTGCGTGTCAT-3'; MYC forward, 5'-CAC TAACATCCCACGCTCTGA-3' and reverse, 5'-AAATCA TCGCAGGCGGAACA-3'; and GAPDH forward, 5'-GCA CCGTCAAGGCTGAGAAC-3' and reverse, 5'-TGGTGAAGA CGCCAGTGGA-3'.

Western blotting. Total protein was extracted form cells using RIPA lysis buffer (Thermo Fisher Scientific, Inc.), according to the manufacturer's protocol. Total protein was quantified using a bicinchoninic acid assay kit (Beijing Solarbio Science \& Technology Co., Ltd.) and $20 \mu \mathrm{g}$ protein/lane was separated via $10 \%$ SDS-PAGE (100 V; $90 \mathrm{~min})$. The separated proteins were subsequently transferred to a polyvinylidene fluoride membrane $(80 \mathrm{~V}$; $90 \mathrm{~min})$ and blocked with $5 \%$ skim milk powder for $1 \mathrm{~h}$ at room temperature.
The membranes were incubated with the following primary antibodies for $12 \mathrm{~h}$ at $4^{\circ} \mathrm{C}$ : Anti-Myc (1:1,000; cat. no. sc-41; Santa Cruz Biotechnology, Inc.) and anti-GAPDH (1:5,000; cat. no. 5174; Cell Signaling Technology, Inc.). Following the primary antibody incubation, a horseradish peroxidase-conjugated goat anti-rabbit IgG secondary antibody (1:5,000; cat. no. ab7090; Abcam) was incubated with the membrane for $1 \mathrm{~h}$ at room temperature. Protein bands were visualized using an ECL detection substrate (Thermo Fisher Scientific, Inc.).

Statistical analysis. Statistical analysis was performed using SPSS version 20.0 software (IBM Corp.) and data are presented as the mean \pm SD of three independent experimental repeats. Statistical differences amongst 2 groups were determined using a Student's t-test, whereas statistical differences between $>2$ groups were determined using one-way ANOVA followed by Tukey's post hoc test for multiple comparisons. Tumor tissues were divided into high expression and low expression groups based on the median value of circ_0067934. The association between clinicopathological features and circ_0067934 expression levels in the 54 bladder cancer tissues were analyzed using a $\chi^{2}$-test. Kaplan-Meier curves were used to analyze the survival rate and a log-rank test was used to calculate the corresponding $\mathrm{P}$-values. $\mathrm{P}<0.05$ was considered to indicate a statistically significant difference.

\section{Results}

High circ_0067934 expression levels in patients with bladder cancer is associated with metastasis, and a low 5-year overall survival and disease-free survival. Circ_0067934 was discovered to be aberrantly expressed in bladder cancer. Significantly increased expression levels of circ_0067934 were found in tumor tissues compared with adjacent normal tissues $(\mathrm{P}<0.001$; Fig. 1A). Notably, patients exhibiting metastasis $(n=34)$ had significantly increased expression levels of circ_0067934 in their tumor tissues compared with patients without metastasis $(\mathrm{n}=20 ; \mathrm{P}<0.001$; Fig. $1 \mathrm{~B})$. In vitro studies also demonstrated that significantly increased circ_0067934 expression levels were found in bladder cancer cells (T24, RT4 and UMUC3) compared with the human bladder epithelial SV-HUC-1 cells $(\mathrm{P}<0.05, \mathrm{P}<0.01$; Fig. $1 \mathrm{C})$. According to the follow-up statistics, patients with high circ_0067934 expression levels $(n=28)$ exhibited a significantly reduced 5-year overall survival and disease-free survival compared with patients with low circ_0067934 expression $(\mathrm{n}=26 ; \mathrm{P}<0.05$; Fig. 1D and E). Notably, it was indicated that circ_0067934 expression was positively correlated with tumor size, advanced stage and lymph node metastasis (Table I).

Reduced circ_0067934 expression levels attenuate the proliferation, migration and invasion of bladder cancer cells. Circ_0067934 exhibited the highest expression in T24 cells (Fig. 1C). Therefore, T24 cells were selected for the following experiments. Circ_0067934 expression levels in T24 cells were significantly reduced following the transfection with sicirc_0067934 compared with siNC-transfected cells $(\mathrm{P}<0.01$; Fig. 2A). The effect of circ_0067934 expression levels on T24 cell proliferation, migration and invasion was analyzed using 
A

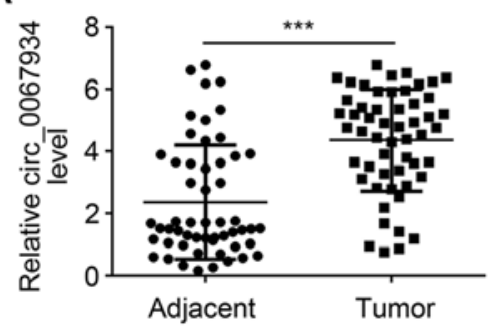

$\mathrm{D}$



B
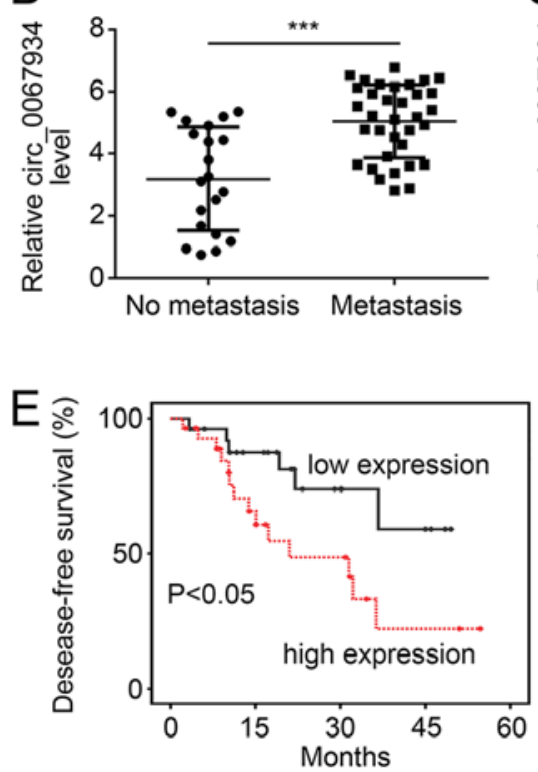

Figure 1. High circ_0067934 expression levels in patients with bladder cancer are associated with metastasis, and a low overall 5-year survival and disease-free survival. (A) circ_0067934 expression levels were significantly increased in bladder cancer tumor tissues compared with the adjacent normal tissues, which were determined using RT-qPCR. (B) Patients with metastasis had significantly increased expression levels of circ_0067934 in their tumor tissues compared with patients without metastasis, which were determined using RT-qPCR. (C) In vitro studies found increased circ_0067934 expression levels in bladder cancer cells (T24, RT4 and UM-UC-3) compared with human bladder epithelial SV-HUC-1 cells, which were determined using RT-qPCR. Patients with high circ_0067934 expression levels exhibited significantly reduced (D) 5-year overall survival and (E) disease-free survival compared with patients with low circ_0067934 expression levels. ${ }^{*} \mathrm{P}<0.05,{ }^{* *} \mathrm{P}<0.01,{ }^{* * *} \mathrm{P}<0.001$. circ, circular RNA; RT-qPCR, reverse transcription-quantitative PCR.
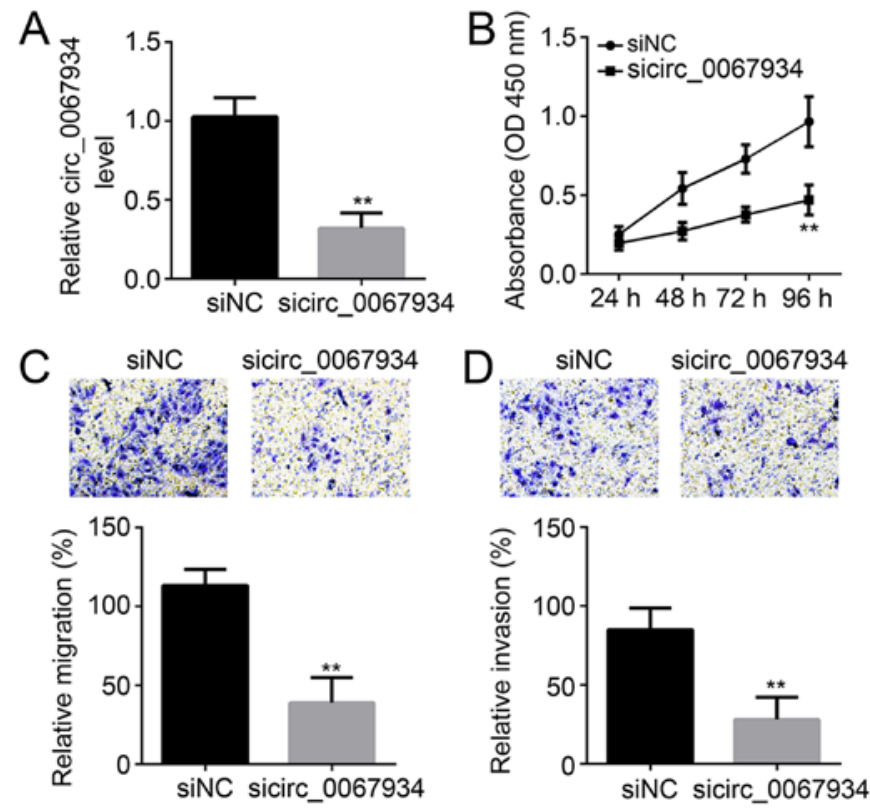

Figure 2. Reduced circ_0067934 expression levels decrease the proliferation migration and invasion of bladder cancer cells. (A) circ_0067934 expression levels in T24 cells were significantly reduced following transfection with sicirc_0067934, which was determined using reverse transcription-quantitative PCR. (B) CCK-8 assay indicated that the knockdown of circ_0067934 significantly reduced the proliferative ability of T24 cells compared with the siNC group. Reduced circ_0067934 expression levels suppressed the (C) migratory and (D) invasive abilities of T4 cells compared with the siNC group (magnification, $\mathrm{x} 100) .{ }^{* *} \mathrm{P}<0.01$ vs. siNC. circ, circular RNA; si, small interfering RNA; NC, negative control; CCK-8, Cell Counting Kit-8; OD, optical density.

the following three methods, respectively: CCK-8, Transwell and Matrigel assays. T24 cells in the sicirc_0067934 group demonstrated a significantly reduced proliferative capacity compared with the siNC-transfected cells ( $\mathrm{P}<0.01$; Fig. $2 \mathrm{~B})$. Similarly, the results of the Transwell and Matrigel assays also revealed that, compared with the siNC group, T24 cells in the sicirc_0067934 group had significantly fewer migratory and invasive cells $(\mathrm{P}<0.01$; Fig. $2 \mathrm{C}$ and $\mathrm{D})$. These findings suggested that the knockdown of circ_0067934 may significantly reduce the proliferative, migratory and invasive abilities of T24 cells.

Circ_0067934 knockdown directly increases miR-1304 expression levels in T24 cells. The WT sequence and Mut sequence of circ_0067934 were designed according to the binding site identified between circ_0067934 and miR-1304, which was predicted using online prediction software (Fig. 3A). T24 cells were transfected with a miR-1304 mimic to overexpress miR-1304 expression; T24 cells in the miR-1304 mimic group demonstrated significantly increased expression levels of miR-1304 compared with the miR-NC mimic group (Fig. 3B). Subsequently, a dual-luciferase reporter assay was performed to validate the relationship between circ_0067934 and miR-1304. For T24 cells in the miR-NC mimic and miR-1304 mimic group, the insertion of the circ_0067934-Mut sequence did not significantly affect the relative luciferase activity between the two groups (Fig. 3C). However, following the circ_0067934-WT sequence being inserted, T24 cells in the miR-1304 mimic group were observed to have a significantly decreased relative luciferase activity compared with the miR-NC mimic group (Fig. 3C), which indicated that circ_0067934 may directly inhibit the expression levels of miR-1304. Concurrently, significantly increased miR-1304 expression levels were observed in T24 cells in the 
B circ_0067934-WT 5'-CGAUUGGGUUGUCAUCCUCAAA-3' miR-1304 3'-GUGUAGAgUgacAuCGgaguUU -5' circ_0067934-Mut 5'-CGAUUGGGUUGUCAUGGAGUUU-3'
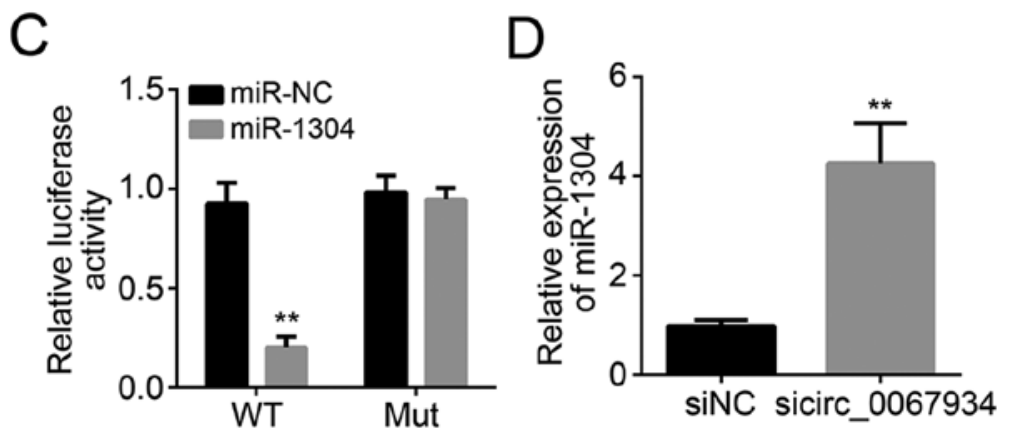
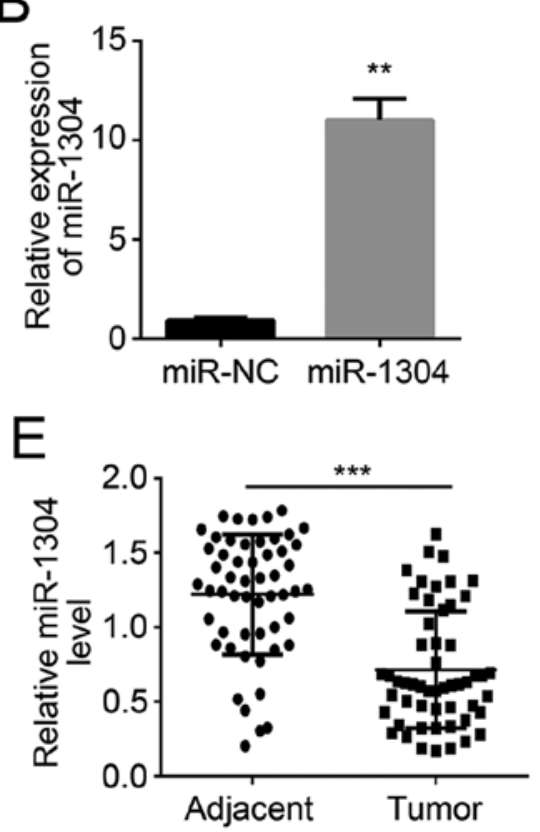

Figure 3. circ_0067934 knockdown directly increases miR-1304 expression in T24 cells. (A) WT and Mut sequences of circ_0067934 were designed according to the binding site identified between circ_0067934 and miR-1304. (B) Relative expression levels of miR-1304 following the transfection of T24 cells with miR-1304 mimic or miR-NC mimic were determined using RT-qPCR. (C) Dual-luciferase reporter assay indicated that circ_0067934 directly inhibited the expression levels of miR-1304. (D) Relative expression levels of miR-1304 were determined using RT-qPCR and significantly increased miR-1304 relative expression levels were observed in T24 cells in the sicirc_0067934 group compared with the siNC group. (E) RT-qPCR analysis was used to determine that the tumor tissues of patients also exhibited significantly decreased miR-1304 expression levels compared with adjacent normal tissues. ${ }^{* *} \mathrm{P}<0.01,{ }^{* * *} \mathrm{P}<0.001$ vs. miR-NC mimic/siNC or adjacent tumor. circ, circular RNA; miR, microRNA; si, small interfering RNA; NC, negative control; RT-qPCR, reverse transcription-quantitative PCR; WT, wild-type; Mut, mutant.

sicirc_0067934 group compared with the siNC group $(\mathrm{P}<0.01$; Fig. 3D). Tumor tissues of patients were also revealed to have significantly decreased miR-1304 expression levels compared with the adjacent normal tissues $(\mathrm{P}<0.001$; Fig. $3 \mathrm{E})$. All of these findings suggested that circ_0067934 may have a direct inhibitory effect over miR-1304 expression and circ_0067934 knockdown may directly promote miR-1304 expression in T24 cells.

miR-1304 directly inhibits Myc expression levels. The binding site between Myc and miR-1304 was predicted using the TargetScanHuman online database, and Myc-WT and Myc-Mut sequences were designed according to the binding site (Fig. 4A). Subsequently, using a dual-luciferase reporter assay, it was discovered that the difference in the relative luciferase activity between the miR-NC mimic group and miR-1304 mimic group was not significant in cells with the Myc-Mut sequence inserted (Fig. 4B). However, the insertion of the Myc-WT sequence significantly decreased the relative luciferase activity of T24 cells in the miR-1304 mimic group compared with the miR-NC mimic group $(\mathrm{P}<0.01$; Fig. 4B). In addition, Myc mRNA and protein expression levels in T24 cells in the miR-1304 mimic group were also decreased compared with the miR-NC mimic group (mRNA, $\mathrm{P}<0.01$; Fig. 4C and D). Similarly, in patients' tissues it was revealed that compared with the adjacent normal tissues, Myc mRNA expression levels in the tumor tissue were significantly increased $(\mathrm{P}<0.001 ;$ Fig. $4 \mathrm{E})$. Therefore, these findings suggested that Myc expression may be directly inhibited by miR-1304.
Circ_0067934 promotes Myc expression through inhibiting miR-1304 expression. T24 cells were transfected with a miR-1304 inhibitor to decrease miR-1304 expression; T24 cells in the miR-1304 inhibitor group demonstrated significantly decreased expression levels of miR-1304 compared with the miR-NC inhibitor group (Fig. 5A). Subsequently, Myc expression levels in T24 cells in the siNC + miR-NC inhibitor group, sicirc_0067934 + miR-NC inhibitor group and sicirc_0067934 + miR-1304 inhibitor group were analyzed. Compared with the siNC + miR-NC inhibitor group, decreased expression levels of Myc mRNA and protein were observed in T24 cells in the sicirc_0067934 + miR-NC inhibitor group (mRNA, P<0.01; Fig. 5B and C); however, compared with the sicirc_0067934 + miR-NC inhibitor group, Myc mRNA and protein expression levels were increased in T24 cells in the sicirc_0067934 + miR-1304 inhibitor group (Fig. 5B and C). Thus, it was suggested that circ_0067934 may promote Myc expression by inhibiting miR-1304.

MYC restoration rescue the effects of circ_0067934 knockdown on $T 24$ cell proliferation, migration and invasion. Significantly increased Myc mRNA expression levels were observed in the oeMyc group compared with the oeVector group $(\mathrm{P}<0.01$; Fig. 6A), indicating that Myc expression in T24 cells was effectively overexpressed following the transfection with the oeMyc. A significantly reduced proliferation and decreased the numbers of migratory and invasive cells were observed in T24 cells in the sicirc_0067934 group + oeVector group compared with the siNC group + oeVector group $(\mathrm{P}<0.01$; Fig. 6B-D). Notably, 
A

Myc-WT 3' UTR 5'-GCCAUAAUGUAAACUGCCUCAAA-3' miR-1304 3'-GUGUAGAGUGACAUCGGAGUUU-5'

Myc-Mut 3' UTR 5'-GCCAUAAUGUAAACUCGGAGUUU-3'
B
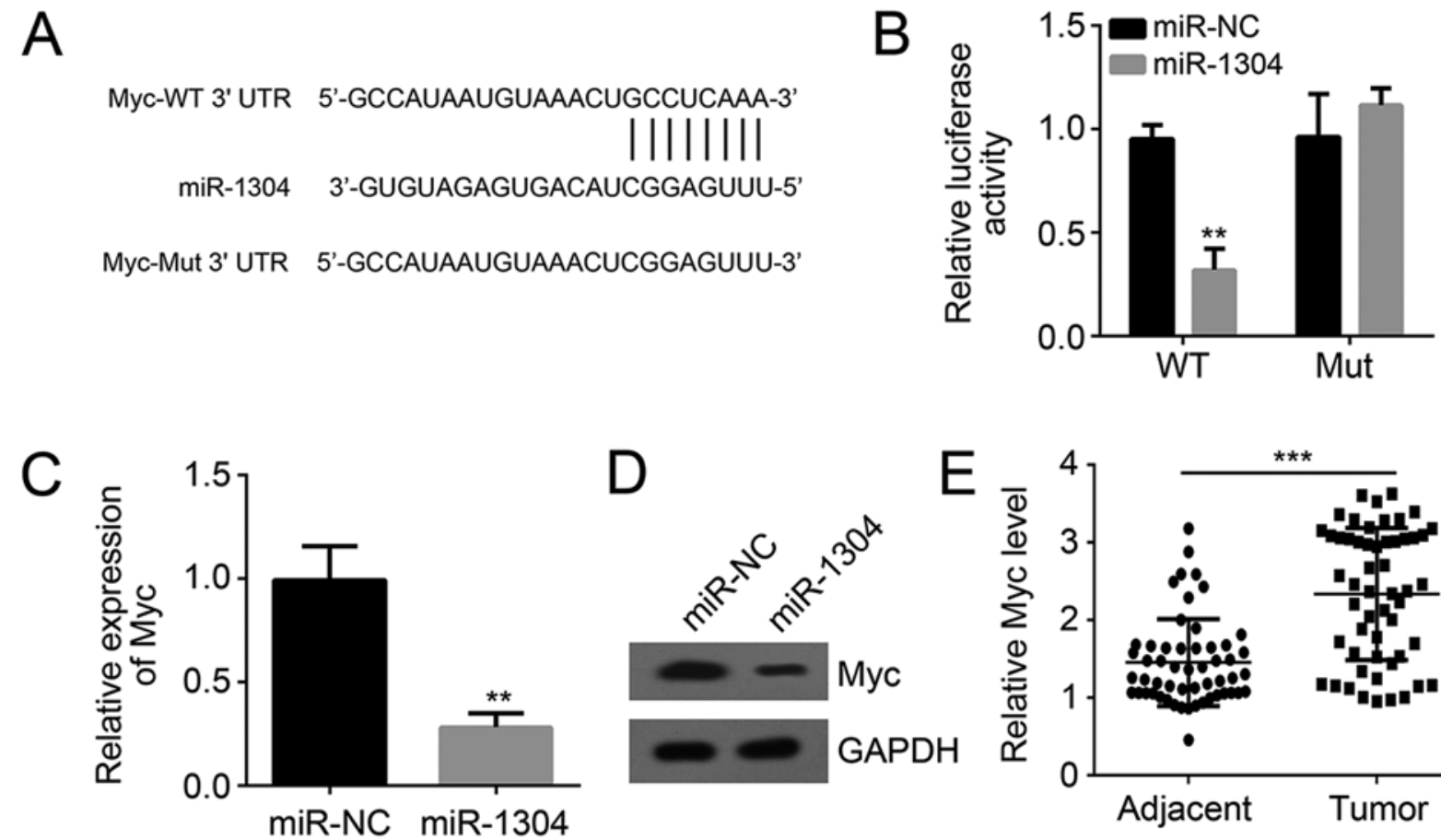
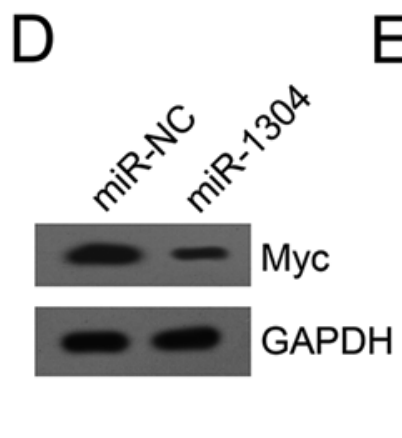

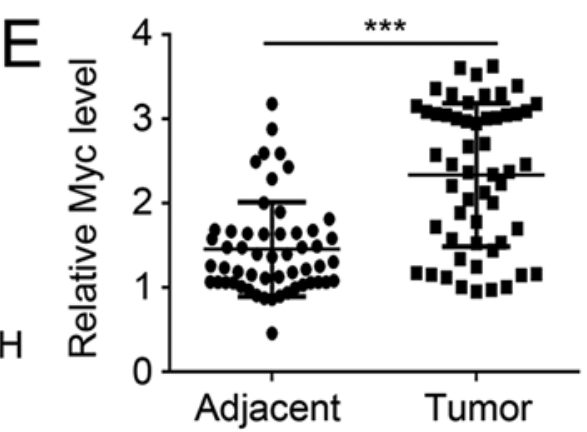

Figure 4. miR-1304 directly inhibits Myc expression. (A) Myc-WT and Myc-Mut sequences were designed according to the binding site identified between miR-1304 and Myc. (B) Dual-luciferase reporter assay revealed that Myc was directly inhibited by miR-1304. Myc (C) mRNA and (D) protein expression levels in T24 cell in the miR-1304 mimic group were decreased compared with the miR-NC mimic group, which was determined using RT-qPCR and western blotting, respectively. (E) RT-qPCR analysis determined that compared with the adjacent normal tissues, Myc mRNA expression levels in the tumor tissues were significantly increased. ${ }^{* *} \mathrm{P}<0.01,{ }^{* * *} \mathrm{P}<0.001$ vs. miR-NC mimic or adjacent tumor. NC, negative control; RT-qPCR, reverse transcription-quantitative PCR; UTR, untranslated region; WT, wild-type; Mut, mutant; miR, microRNA.


Figure 5. circ_0067934 promotes Myc expression via inhibiting miR-1304. (A) Relative expression levels of miR-1304 following the transfection with the miR-1304 inhibitor or miR-NC inhibitor were determined using RT-qPCR. T24 cells in the sicirc_0067934 + miR-NC inhibitor group exhibited decreased Myc (B) mRNA and (C) protein expression levels compared with the siNC + miR-NC inhibitor group, whereas Myc expression levels were restored in the sicirc_0067934 + miR-1304 inhibitor group, which was determined using RT-qPCR and western blotting, respectively. ${ }^{* *} \mathrm{P}<0.01 \mathrm{vs}$. siNC + NC inhibitor. circ, circular RNA; miR, microRNA; si, small interfering RNA; NC, negative control; RT-qPCR, reverse transcription-quantitative PCR.

based on the results of the CCK-8 and Transwell assays, T24 cells in the sicirc_0067934 + oeMYC group also rescued the abilities of proliferation, migration and invasion compared with T24 cells in the sicirc_0067934 + oeVector group ( $\mathrm{P}<0.01$; Fig. 6B-D). Furthermore, The proliferation, migration and invasion of T24 cells in the siNC + oeMYC group were further enhanced compared with the sicirc_0067934 + oeMYC group (Fig. 6B-D). MYC overexpression reversed the effects of circ_0067934 silencing on T24 cells.

\section{Discussion}

Bladder cancer is the most common malignant tumor of the genitourinary system, and can be divided into myometrial invasive bladder cancer and non-muscle invasive bladder cancer according to the histopathological features (15). According to reports, $75 \%$ of patients with bladder cancer have non-muscle invasive bladder cancer, and $70 \%$ of these will subsequently relapse, with $15 \%$ progressing to myometrial invasive bladder cancer (16). Myometrial invasive bladder 



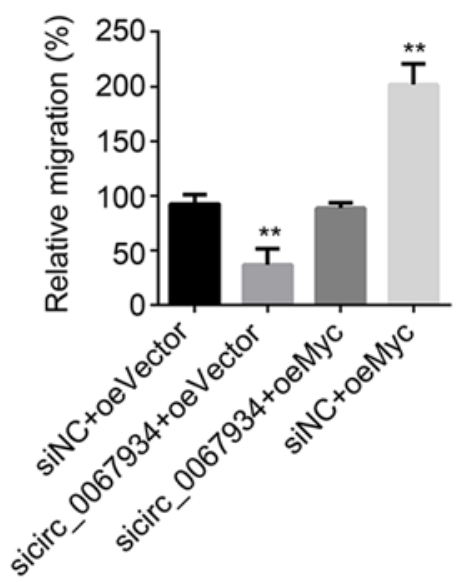

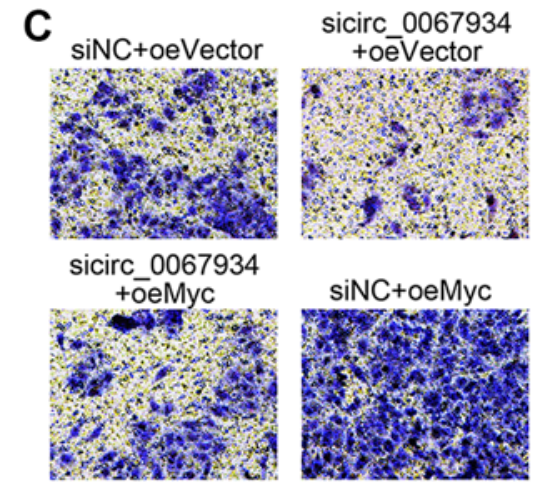

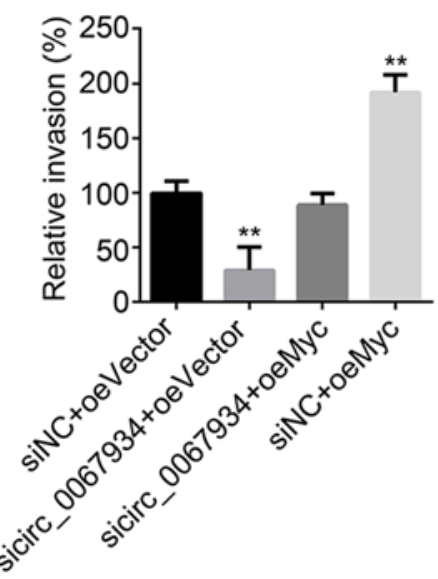

Figure 6. circ_0067934 knockdown suppresses T24 cell proliferation, migration and invasion through inhibiting Myc expression. (A) Reverse transcription-quantitative PCR analysis demonstrated that Myc expression in T24 cells was significantly increased following the transfection with the oeMyc compared with the oeVector. (B) Transwell and (C) Matrigel assays were used for the analysis of migration and invasion, respectively (magnification, x100). (D) Proliferative activity of T24 cells was measured using a CCK-8 assay. ${ }^{* *} \mathrm{P}<0.01$ vs. siNC + oeVector. circ, circular RNA; si, small interfering RNA; NC, negative control; CCK-8, Cell Counting Kit-8; OD, optical density; oe, overexpression.

cancer is more aggressive and $50 \%$ of these patients have been reported to exhibit distant metastasis, with a 5-year survival rate of only $50 \%$ (17). Despite the rapid development of surgical and early diagnosis techniques, the mortality rate of patients with bladder cancer has not decreased significantly in the past 20 years (18). Therefore, determining the molecular mechanisms of bladder cancer development is particularly important for discovering novel diagnostic markers, and for developing effective and individualized treatment options. The present study identified that circ_0067934 expression levels were significantly increased in bladder cancer and the knockdown of circ_0067934 suppressed bladder cancer cell proliferation, migration and invasion though inhibiting the expression of Myc. And circ_0067934 expression was positively correlated with tumor size, advanced stage and lymph node metastasis

Circ_0067934 is a circRNA which has been reported to be abnormally expressed in several types of human tumor (19). Previous studies have discovered that circ_0067934 expression was significantly increased in non-small cell lung cancer; these increased expression levels in tumor tissues were closely associated with a poor prognosis of patients with non-small cell lung cancer, alongside an advanced tumor-node-metastasis (TNM) stage, positive lymph node metastasis, distant metastasis and poor survival (19). In fact, circ_0067934 has been suggested to serve as a therapeutic target and a biomarker to evaluate the prognosis of patients with non-small cell lung cancer, which was discovered following the reduced proliferative, migratory and invasive abilities observed following the silencing of circ_0067934 in non-small cell lung cancer cells (19). Furthermore, a cohort study of 51 esophageal squamous cell carcinoma cases indicated that the expression levels of circ_0067934 in esophageal squamous cell carcinoma were markedly increased compared with adjacent normal tissues (12). Increased circ_0067934 expression levels were also associated with an advanced TNM stage and poor differentiation (12). In the present study, circ_0067934 was discovered to be abnormally overexpressed in bladder cancer tissues and cells, and high circ_0067934 expression levels were associated with metastasis, and a low 5-year overall and disease-free survival. In addition, it was suggested that circ_0067934 may be a therapeutic target and prognostic marker for bladder cancer, as the silencing of circ_0067934 inhibited the proliferative, migratory and invasive ability of bladder cancer cells. Notably, several other circRNAs have been reported to regulate bladder cancer, such as hsa_circ_0068871 and circRNA lysophosphatidic acid receptor $1(20,21)$. Whether circ_0067934 expression is correlated with the expression of these reported circRNAs requires further investigation.

One of the most important functions of circRNAs is to serve as an miRNA sponge (22). CircRNAs can competitively bind to miRNAs to inhibit the binding of miRNAs to target genes, thereby blocking miRNA function $(7,23)$. Zhu et al (13) observed that circ_0067934 was overexpressed in hepatocellular carcinoma and this directly inhibited the expression of 
miR-1324, which could target the 3'-UTR of frizzled-5 mRNA and further suppress the $\mathrm{Wnt} / \beta$-catenin signaling pathway to inhibit hepatocellular carcinoma progression. In the present study, it was confirmed that circ_0067934 promoted the proliferation, migration and invasion of bladder cancer cells by promoting Myc expression via sponging miR-1304. In a previous study, miR-1304 was demonstrated to inhibit the growth of human non-small cell lung cancer cells, thus serving as a tumor suppressor (24). Myc protein has also been found to have a crucial role in several important cellular processes, such as proliferation, apoptosis, differentiation and the cell cycle, and overall it has been reported to serve a major role in driving the proliferation of cancer cells (25). Previous studies have also pointed out that the suppression of Myc could contribute to tumor regression (26). Myc is found activated in a wide variety of tumors, thus making it an attractive target for cancer treatment. The findings of the current study demonstrated that the inhibition of circ_0067934 prevented bladder cancer progression by suppressing the expression of Myc through sponging miR-1304, which provided a more precise theoretical basis for targeted therapy in bladder cancer.

In conclusion, the present study revealed that increased circ_0067934 expression levels in bladder cancer were associated with a poor prognosis in patients. In addition, silencing circ_0067934 expression prevented the proliferative, migratory and invasive abilities of bladder cancer cells through inhibiting the expression levels of Myc protein via sponging miR-1304. Thus, these findings suggested that circ_0067934 may be used as a potential biomarker for assessing the prognosis of patients with bladder cancer and as a target for the treatment of bladder cancer.

\section{Acknowledgements}

Not applicable.

\section{Funding}

No funding was received.

\section{Availability of data and materials}

All data generated and/or analyzed during the present study are included in this published article.

\section{Authors' contributions}

QL and PZ conceived and designed the present study. QL and QZ performed the experiments. PZ analyzed and interpreted the results and wrote the manuscript. All authors read and approved the final manuscript.

\section{Ethics approval and consent to participate}

The present study was approved by The Ethics Committee of Hanchuan People's Hospital. Written informed consent was obtained from all enrolled patients.

\section{Patient consent for publication}

Not applicable.

\section{Competing interests}

The authors declare that they have no competing interests.

\section{References}

1. Liu Z and Zhang H: LncRNA plasmacytoma variant translocation 1 is an oncogene in bladder urothelial carcinoma. Oncotarget 8: 64273-64282, 2017.

2. Gayyed MF and Tawfiek ER: Utility of SOX2 and livin co-expression in the prognosis of bladder cancer with bilharzial and non-bilharzial bladder status. World J Oncol 6: 446-455, 2015.

3. Iyer MK, Niknafs YS, Malik R, Singhal U, Sahu A, Hosono Y, Barrette TR, Prensner JR, Evans JR, Zhao S, et al: The landscape of long noncoding RNAs in the human transcriptome. Nature Genet 47: 199-208, 2015.

4. Xu XW, Zheng BA, Hu ZM, Qian ZY, Huang CJ, Liu XQ and Wu WD: Circular RNA hsa_circ_000984 promotes colon cancer growth and metastasis by sponging miR-106b. Oncotarget 8: 91674-91683, 2017.

5. Xu T, Wu J, Han P, Zhao Z and Song X: Circular RNA expression profiles and features in human tissues: A study using RNA-seq data. BMC Genomics 18 (Suppl): 680, 2017.

6. Quan G and Li J: Circular RNAs: Biogenesis, expression and their potential roles in reproduction. J Ovarian Res 11: 9, 2018.

7. Lyu D and Huang S: The emerging role and clinical implication of human exonic circular RNA. Rna Biol 14: 00-00, 2016.

8. Zheng Q, Bao C, Guo W, Li S, Chen J, Chen B, Luo Y, Lyu D, Li Y, Shi G, et al: Circular RNA profiling reveals an abundant circHIPK 3 that regulates cell growth by sponging multiple miRNAs. Nat Commun 7: 11215, 2016.

9. Zhong Z, Huang M, Lv M, He Y, Duan C, Zhang L and Chen J: Circular RNA MYLK as a competing endogenous RNA promotes bladder cancer progression through modulating VEGFA/VEGFR2 signaling pathway. Cancer Lett 403: 305, 2017.

10. Li P, Yang X, Yuan W, Yang C, Zhang X, Han J, Wang J, Deng X, Yang H, Li P, et al: CircRNA-cdrlas exerts anti-oncogenic functions in bladder cancer by sponging MicroRNA-135a. Cell Physiol Biochem 46: 1606-1616, 2018.

11. Li Y, Zheng F, Xiao X, Xie F, Tao D, Huang C, Liu D, Wang M, Wang L, Zeng F and Jiang G: CircHIPK3 sponges miR-558 to suppress heparanase expression in bladder cancer cells. EMBO Rep 18: 1646-1659, 2017.

12. Xia W, Qiu M, Chen R, Wang S, Leng X, Wang J, Xu Y, Hu J, Dong G, Xu PL and Yin R: Circular RNA has_circ_0067934 is upregulated in esophageal squamous cell carcinoma and promoted proliferation. Sci Rep 6: 35576, 2016.

13. Zhu Q, Lu G, Luo Z, Gui F, Wu J, Zhang D and Ni Y: CircRNA circ_0067934 promotes tumor growth and metastasis in hepatocellular carcinoma through regulation of miR-1324/FZD5/Wnt/ $\beta$-catenin axis. Biochem Biophys Res Commun 497: 626-632, 2018.

14. Livak KJ and Schmittgen TD: Analysis of relative gene expression data using real-time quantitative PCR and the 2(-Delta Delta C(T)) method. Methods 25: 402-408, 2001.

15. Svatek RS, Hollenbeck BK, Sten HN, Richard L, Kim SP Arnulf $\mathrm{S}$ and Yair L: The economics of bladder cancer: Costs and considerations of caring for this disease. Eur Urol 66: 253-262, 2014.

16. Jr GRP, Friedell GH, Culp DA, Loening S, Cummings KB Cutler SJ, Flanagan MJ, Jr WWK, Pearse $\mathrm{H}$ and Merrin C: Superficial bladder cancer: Progression and recurrence. J Urology 130: 1083-1086, 1983.

17. Wang T, Yuan J, Feng N, Li Y, Lin Z, Jiang Z and Gui Y: Hsa-miR-1 downregulates long non-coding RNA urothelial cancer associated 1 in bladder cancer. Tumor Biol 35: 10075-10084, 2014.

18. Rosser CJ, Urquidi V and Goodison S: Urinary biomarkers of bladder cancer: An update and future perspectives. Biomark Med 7: 779-790, 2013.

19. Wang J and Li H: CircRNA circ_0067934 silencing inhibits the proliferation, migration and invasion of NSCLC cells and correlates with unfavorable prognosis in NSCLC. Eur Rev Med Pharmacol Sci 3053-3060, 2018. 
20. Mao W, Huang X, Wang L, Zhang Z, Liu M, Li Y, Luo M Yao X, Fan J and Geng J: Circular RNA hsa_circ_0068871 regulates FGFR3 expression and activates STAT3 by targeting miR-181a-5p to promote bladder cancer progression. J Exp Clin Cancer Res 38: 169, 2019.

21. Lin G, Sheng H, Xie H, Zheng Q, Shen Y, Shi G and Ye D: circLPAR1 is a novel biomarker of prognosis for muscle-invasive bladder cancer with invasion and metastasis by miR-762. Oncol Lett 17: 3537-3547, 2019.

22. Hansen TB, Jensen TI, Clausen BH, Bramsen JB, Finsen B, Damgaard CK and Kiems J: Natural RNA circles function as efficient microRNA sponges. Nature 495: 384-388, 2013.

23. Bonizzato A, Gaffo E, Te Kronnie G and Bortoluzzi S: CircRNAs in hematopoiesis and hematological malignancies. Blood Cancer J 6: e483, 2016.
24. Li CG, Pu MF, Li CZ, Gao M, Liu MX, Yu CZ, Yan H, Peng C, Zhao Y, Li Y, et al: MicroRNA-1304 suppresses human non-small cell lung cancer cell growth in vitro by targeting heme oxygenase-1. Acta Pharmacol Sin 38: 110-119, 2017.

25. Posternak V and Cole MD: Strategically targeting MYC in cancer. F1000Res 5: pii: F408, 2016.

26. Felsher DW: MYC inactivation elicits oncogene addiction through both tumor cell-intrinsic and host-dependent mechanisms. Genes Cancer 1: 597-604, 2010.

This work is licensed under a Creative Commons Attribution-NonCommercial-NoDerivatives 4.0 International (CC BY-NC-ND 4.0) License. 